\title{
FUTURE AGN RESEARCH II
}

\author{
GREGORY A. SHIELDS \\ Department of Astronomy \\ University of Texas at Austin \\ Austin, Texas 78712
}

\begin{abstract}
The "standard model" of AGN involves a supermassive black hole accreting gas, possibly through a flattened disk. This model enjoys widespread popularity among astronomers, but convincing proof has been elusive. Recent observations of "dormant" black holes in nearby galactic nuclei encourage the idea that black holes lie at the centers of AGN. An understanding of the nature of the accretion flow seems most likely to come from Doppler shifts of spectral features. The thermal continuum from the inner disk offers an intriguing opportunity. Theoreticians should not neglect the study of the thin disks that should exist for some AGN parameters, as these relatively "tame" objects may yield the most reliable observational tests. The broad emission lines may represent the debris of tidally disrupted stars.
\end{abstract}

\section{Dormant Black Holes in Galactic Nuclei}

Are supermassive black holes really present in galactic nuclei? How fast do they accrete gas? Does the accretion flow look like an AGN? These questions have been central to AGN research for two decades (e.g., Lynden-Bell 1969), but today they cannot be answered with certainty. Future research must largely be aimed at finding the answers.

Progress on the first question has been achieved through spectroscopic observations of nearby galactic nuclei at high spatial resolution. Alan Dressler describes in this volume evidence for central black holes of mass $\sim 10^{7} \mathrm{M}_{\odot}$ in the nuclei of the nearby spiral M 31 and elliptical M 32 (Tonry 1984; Dressler and Richstone 1988; Kormendy 1988). Dynamics of clouds near the center of our Galaxy suggest a black hole of mass $\sim 10^{6.5} \mathrm{M}_{\odot}$ (Serabyn et al. 1988).

The apparent prevalence of moderate size black holes in nearby nuclei raises questions concerning what energetic phenomena might be manifested in the absence of full fledged AGN activity. Fabian and Canizares (1988) argue that a black hole should accrete the hot interstellar gas that is revealed by X-ray observations in the nuclei of elliptical galaxies. If the calculated accretion rate $\dot{\mathrm{M}}$ produces a luminosity $\mathrm{L} \simeq 0.1 \dot{\mathrm{M}} \mathrm{c}^{2}$, then the absence of observed nuclear activity in nearby ellipticals implies upper limits on the masses of black holes present. The inferred limits may conflict with statistical predictions of the prevalence of "burned out" black holes by Soltan (1982). Are the calculated accretion rates inevitable, and is an inefficient "ion torus" (Begelman 1985) an alternative to the assumed $\sim 0.1 c^{2}$ efficiency? The example of M 87 is suggestive. The nuclear luminosity is much lower than might be expected if the X-ray inferred 
cooling flow of $\sim 3 \mathrm{M}_{\odot} \mathrm{yr}^{-1}$ (Fabricant and Gorenstein 1987) ultimately falls into a black hole at $0.1 \mathrm{c}^{2}$ efficiency.

Black holes in galactic nuclei are surrounded by stars, and occasional tidal disruptions are inevitable (Hills 1975). Rees (1988) argues that in relatively quiescent nuclei such as M 31 or the Galactic center, disruptions might occur at intervals of $\sim 10^{4}$ years. Half of the debris would soon settle into a tightly bound torus, which might quickly and inefficiently accrete onto the hole. The resulting flare would shine at roughly the hole's Eddington luminosity, but with a brief duty cycle of only $\sim 10^{-3}$. An important insight is the realization (Lacy, Townes, and Hollenbach 1982; Rees 1988) that roughly half the debris is ejected at speeds up to $\sim 10^{4} \mathrm{~km} \mathrm{~s}^{-1}$. Thus, tidal disruption models of the AGN accretion supply predict roughly equal amounts of accreted and ejected gas, and the effects of the energy, momentum, and mass of the ejected gas are interesting topics for study.

\section{Active Black Holes}

The cataclysmic variable stars resemble the standard model of AGN in having accretion disks around compact objects. Why are we much more certain of the basic geometry of cataclysmic variables than that of AGN? The answer involves the dynamical information provided by Doppler shifts and periodic variations associated with the orbital motion. Unless the suggestion by Gaskell (1988) of binary black holes in AGN is borne out, we are deprived of these opportunities in AGN. Nevertheless, the basic question is a dynamical one: does gas moving at high speeds near black holes provide the power for AGN? This suggests that the most convincing observations will involve direct measurements of the orbital or infall motion, most likely by means of Doppler shifts of spectral features.

\subsection{THE BROAD EMISSION LINES}

Broad emission lines with widths $\sim 10,000 \mathrm{~km} \mathrm{~s}^{-1}$ are a hallmark of AGN. There is no generally accepted theory of the origin and motion of the photoionized clouds that are believed to produce the broad lines. The dynamics of the broad line region (BLR) is reviewed by Mathews and Capriotti (1985). Broad line profiles, often characterized as "logarithmic," can be reproduced by radiative acceleration or gravitational motion (Mathews and Blumenthal 1977; Capriotti, Folz, and Byard 1980; Hubbard and Puetter 1983). One clue to the kinematical pattern is the lack of strong asymmetry in the optically thick lines such as $\mathrm{L} \alpha$ and $\mathrm{H} \alpha$, which should be stronger in the red wing for outflow and in the blue wing for infall. Kwan and Carroll (1982) emphasized that orbital motion could give symmetrical profiles and illustrated this with a model involving clouds falling near the hole on parabolic orbits. Further support for a model involving orbital motion is provided by the fact that in most cases, the red and blue wings vary with time in a symmetrical fashion (e.g., Ulrich et al. 1984). Bound motions require rather large black holes for the BLR radii implied by photoionization models, but temporal variability studies indicate smaller radii for which the required orbital velocities correspond to smaller masses (Mathews and Capriotti 1985).

An exciting possibility regarding orbital motion of the gas emitting the broad emis- 
sion lines was raised by Halpern and Filippenko (1988). The broad lines of $\mathrm{H} \alpha$ and $\mathrm{H} \beta$ show two peaks almost equally redshifted and blueshifted by $\sim 5000 \mathrm{~km} \mathrm{~s}^{-1}$ with respect to the systemic velocity. This can be fit with a torus of gas in Keplerian motion around a massive black hole (see Halpern and Chen in this volume). The blue wing is brighter than the red wing as expected from relativistic effects, and the line center is redshifted in a way consistent with the gravitational redshift for a disk inclination $\mathrm{i}$ $\approx 30^{\circ}$. This interpretation may not be unique, since the same qualitative features are shown by the high velocity emission lines of SS 433 (e.g., Margon, Grandi, and Downes 1980) attributed to fast moving gas in oppositely directed jets. Moreover, irregular broad line profiles, in particular double peaks, are common in AGN spectra, without the degree of symmetry shown by Arp 102B (Gaskell 1988).

Monitoring the variations of the broad line intensities and profiles in comparison with continuum variations is a powerful technique receiving increasing attention (e.g., Ulrich et al. 1984; Peterson et al. 1985; Gaskell and Sparke 1986). The symposium witnessed a lively debate on the proper way to analyze such measurements (see contributions by Peterson and by Edelson and Krolik in this volume). The arguments for inflow of the broad line gas by Gaskell, Koratkar, and Sparke in this volume are illustrative of the potential of this technique. However, past experience shows that many episodes must be observed before a systematic pattern of variations can be claimed (e.g., Ulrich et al). Thus, a concerted effort to conduct sustained monitoring of a variety of individual objects is needed.

\subsection{TIDAL DISRUPTION AND THE BROAD EMISSION LINES}

Tidal disruption of stars by a supermassive black hole has been considered as a possible source for the accretion supply of AGN (Hills 1975; Shields and Wheeler 1978, and references therein). Disruption requires a low angular momentum orbit with a pericenter inside the tidal radius (Hills 1975),

$$
r_{T}=\left(1.3 \times 10^{13} \mathrm{~cm}\right) M_{6}^{1 / 3}\left(\rho / \rho_{\odot}\right)^{-1 / 3}
$$

where $\mathrm{M}_{6}=\mathrm{M} / 10^{6} \mathrm{M}_{\odot}$ and $\rho$ is the effective stellar density. Sustained rates of tidal disruption may be too low if stellar orbits evolve in angular momentum only on a binary relaxation timescale, but collective effects may enhance orbit diffusion.

Lacy, Townes, and Hollenbach (1982) noted that at the point of disruption, portions of the debris acquire velocities of $\sim v_{*}$ relative to the debris' center of mass, where $v_{*}$ $=\left(2 G M_{*} / r_{*}\right)^{1 / 2}$. This velocity spread, superimposed on the overall orbital velocity $v_{T}$ $=\left(2 G \cdot M / r_{T}\right)^{1 / 2}$, leads to a range in specific binding energies to the hole, from $\mathrm{E}_{\min }$ $\simeq-v_{*} v_{T}$ to $\mathrm{E}_{\max } \simeq v_{*} v_{T}$. Rees (1988) assumes that the debris are uniformly distributed in $\mathrm{E}$ between $\mathrm{E}_{\min }$ and $\mathrm{E}_{\max }$.

Using the solar surface values for $v_{*}$ and $\rho$, we have

$$
v_{\max }=\left(2 v_{T} v_{*}\right)^{1 / 2}=\left(3800 \mathrm{~km} \mathrm{~s}^{-1}\right) M_{6}^{1 / 6} .
$$

The median energy of the unbound debris corresponds to a terminal velocity $2^{-1 / 2}$ $v_{\max }$; and the bound debris will follow highly eccentric orbits with characteristic apoc- 
enter $r_{o}=2 \mathrm{GM} / v_{o}^{2}$, where again $v_{o}=2^{-1 / 2} v_{\max }$. Under the influence of the central ionizing continuum, both the bound and unbound debris will produce emission lines characterized by widths of order $\sim 10^{4} \mathrm{~km} \mathrm{~s}^{-1}$, with only a weak dependence on $\mathrm{M}$. Over several orders of magnitude in luminosity, the observed broad emission lines of AGN have just such widths; and I suggest that they may be emitted by tidal debris.

Let us assume that the tidal debris breaks up into small clouds that eventually dissolve into a hot medium undergoing spherical flow. Much of the mass injection into the medium will occur at radii $\sim r_{o}$; and we may guess that a stagnation radius, also near $r_{o}$, divides the inner zone of infall from the outer zone of outflow, with $\dot{\mathrm{M}}_{\text {in }} \approx \dot{\mathrm{M}}_{\text {out }} \approx$ $\dot{\mathrm{M}} / 2$. The accretion produces a luminosity $L=\epsilon c^{2} \dot{\mathrm{M}}_{i n}$, where $\epsilon \approx 0.1$. In the vicinity of $r_{o}$, the Compton cooling time exceeds the radial flow time, so that the gas remains at roughly the virial temperature, $\mathrm{T} \approx M_{H} v_{o}^{2} / 6 k \approx 10^{9} \mathrm{~K}$. The ionizing radiation flux striking clouds near $r_{o}$ is $\phi=\left(\mathrm{L} / 4 \pi r_{o}^{2}\right)\left(\xi / I_{H}\right)$, where $\xi$ is a continuum shape factor. BLR clouds at temperature $T_{B} \simeq 10^{4} \mathrm{~K}$ in pressure equilibrium with the medium have a density $n_{B}=\left(\dot{\mathrm{M}}_{i n} / 4 \pi r_{o}^{2}\right)\left(v_{m} m_{H}\right)^{-1}\left(c_{m}^{2} / c_{B}^{2}\right)$, where $c_{B}$ and $c_{m}$ are the cloud and medium sound speeds and $v_{m}$ is the medium flow velocity. The ionization parameter for the broad line clouds is

$$
U_{B}=\frac{\phi}{n_{B} c}=\xi \epsilon \frac{m_{H} c^{2}}{I_{H}} \frac{v_{m}}{c} \frac{c_{B}^{2}}{c_{m}^{2}} \simeq 10^{-1.7} v_{m 9}^{-1},
$$

where we have taken $\xi=0.1, \epsilon=0.1$, and $c_{m} \simeq v_{m}=\left(10^{9} \mathrm{~cm} \mathrm{~s}^{-1}\right) v_{m 9}$. For $v_{m 9}$ $\simeq 1$, this agrees with the ionization parameter inferred from photoionization models (see review by Ferland and Shields 1985). The lack of dependence on $M$ and $\dot{M}_{i n}$ is also consistent with the uniformity of line ratios and inferred ionization parameters among Seyfert galaxies and QSOs. The predicted ionization parameter has a fairly weak dependence on radius in a given object, and applies in a first approximation both to bound and unbound debris. McCray (1979) and Krolik and London (1983) also discuss the ionization parameter in the case that the BLR confining pressure is supplied by a spherical accretion flow.

Given the profile symmetry arguments above, we consider models in which the line emission comes mostly from bound clouds that complete one or more elliptical orbits. Suppose that clouds are created near $r_{T}$ with some binding energy $E_{o}$. If they move on essentially radial orbits and emit an isotopic luminosity $\epsilon(\mathrm{r})=\epsilon_{o}\left(r / r_{o}\right)^{a}$ per unit mass in a line at rest frequency $\nu_{o}$, then the time averaged line profile is

$$
L_{\nu}(w)=\frac{\epsilon_{o} m}{P g_{o} \mu}\left(1+\frac{w^{2}}{\mu^{2} v_{o}^{2}}\right)^{-2+a}
$$

where $\mathrm{P}$ is the period, $\mathrm{m}$ the cloud mass, $g_{o}=\mathrm{GM} / r_{o}^{2}, \mu=\cos \theta$, and $w=c \Delta \nu / \nu$ is the line-of-sight velocity. For continuous cloud creation at rate $\dot{M}$, the quantity $m / P$ is replaced by $\dot{M} N_{o r b}$, where $\mathrm{N}_{\text {orb }}$ is the number of orbits completed before cloud destruction. This profile (Fig. 1) already is a tolerable approximation to a typical broad line profile (see Osterbrock 1985, and references therein). If the medium pressure varies as $r^{-3 / 2}$ (isothermal free fall) and the clouds remain roughly spherical, the solid angle of ionizing continuum intercepted by one optically thick cloud varies as $r^{-1}$, so that $a$ 
$=1$. If $c_{m}^{2}$ varies as $r^{-1}$ (virial temperature or ram pressure confinement), then $a=$

$1 / 3$. Shields (1978b) characterized the $\mathrm{H} \alpha$ wings of $3 \mathrm{C} 120$ as $L_{\nu} \propto w^{-5 / 2}$, requiring $a$ $=3 / 4$. A key point is that the line peaks at $w=0$, in contrast to the two-horned profile of gas in circular orbit. Careful tailoring of the emissivity with radius is needed in Keplerian disk models. Furthermore, since the tidal debris comes to rest at $r_{o}$, there is no remote gas to create undesirable broad emission wings of [O III] as occur in $\mathrm{Ke}-$ plerian disk models (Shields 1978b). The extreme wings come from gas at small radii, and Carroll and Kwan (1985) show that this is consistent with comparative profiles of different lines.

Integration of the profiles of equation (4) over $\mu$ gives profiles with logarithmic cores and wings $L_{\nu} \propto w^{-4+2 a}$; and for $\mathrm{a}=1$, one has $L_{\nu}=\ln \left(1+v_{o}^{2} / w^{2}\right)$. The divergence at $w=0$ is easily avoided by noting that the orbits are actually ellipses with transverse velocities $v_{\phi} \simeq 500 \mathrm{~km} \mathrm{~s}^{-1}$ at apocenter. Figure 1 shows a profile $L_{\nu}=\ln \left[1+v_{o}^{2} /\left(w^{2}\right.\right.$ $+v_{\phi}^{2}$, with $v_{\phi}=0.1 v_{o}$. Integration of equation (4) over a range of $v_{o}^{2}$ from $v_{1}^{2}$ to $v_{\max }^{2}$ also gives good profiles. For $a=1$ and fixed $\mu, L_{\nu}=\ln \left[\left(w^{2}+\mu^{2} v_{\max }^{2}\right)\right.$ $\left./\left(w^{2}+\mu^{2} v_{1}^{2}\right)\right]$. For $v_{1}=0.1 v_{\max }$, this is essentially the same profile as the $\mu$ averaged profile shown in Figure 1, aside from a width scale faction $\mu v_{\max } / v_{0}$.

We conclude that tidal disruption gives a natural explanation of observed line widths and a robust explanation of broad line profiles. An exciting prospect is the use of equation (2) to determine black hole masses from line widths. A theoretical calibration of this relation will require a more detailed understanding of tidal disruption and the importance of red giant disruptions. Moreover, the debris in a given AGN may not be isotropically distributed around the hole, so that small values of $\mu$ could give narrow observed profiles. An overall increase of line width with luminosity, $\delta v \propto L^{0.2}$, has been reported (Joly et al. 1985). This is consistent with equation (2) if L increases with $M$ statistically. If broader lines correlate with large $M$, then studies of stellar velocities in nearby AGN should concentrate on those with the broadest emission lines. Relative values of $\mathrm{M}$ derived from line widths could be compared with other indicators as they are developed, such as phenomena depending on $L / L_{E}$.

\subsection{THERMAL CONTINUUM FROM A DISK}

The broad lines receive much attention because of the wealth of spectral information they offer, but they occur relatively far from the black hole, where GM/R $\approx 10^{-4} \mathrm{c}^{2}$. An understanding of AGN structure at GM/Rc $\mathrm{C}^{2} \approx 10^{-1}$, where the maximum power is liberated, is a goal of obvious importance. This region has been the subject of much theoretical effort, but definitive observational evidence has been lacking. Perhaps the most concrete hope for a direct observation of this region is afforded by the interpretation of the blue-ultraviolet continuum "bump" in terms of thermal emission from an accretion disk (Shields 1978a; Malkan and Sargent 1982).

The theory of AGN accretion disks is reviewed by Begelman (1985). The optically thick, relatively cool solutions with $\mathrm{kT}<<\mathrm{GM} m_{H} / R$ fall into the categories of standard thin disks (Shakura and Sunyaev 1973) and thick radiation tori (e.g., Paczynski 1981 , and references therein). Thin accretion disks, for $\mathrm{M} \approx 10^{8} \mathrm{M}_{\odot}$, have maximum effective temperature $\mathrm{T} \approx 10^{5} \mathrm{~K}$ near the hole; 


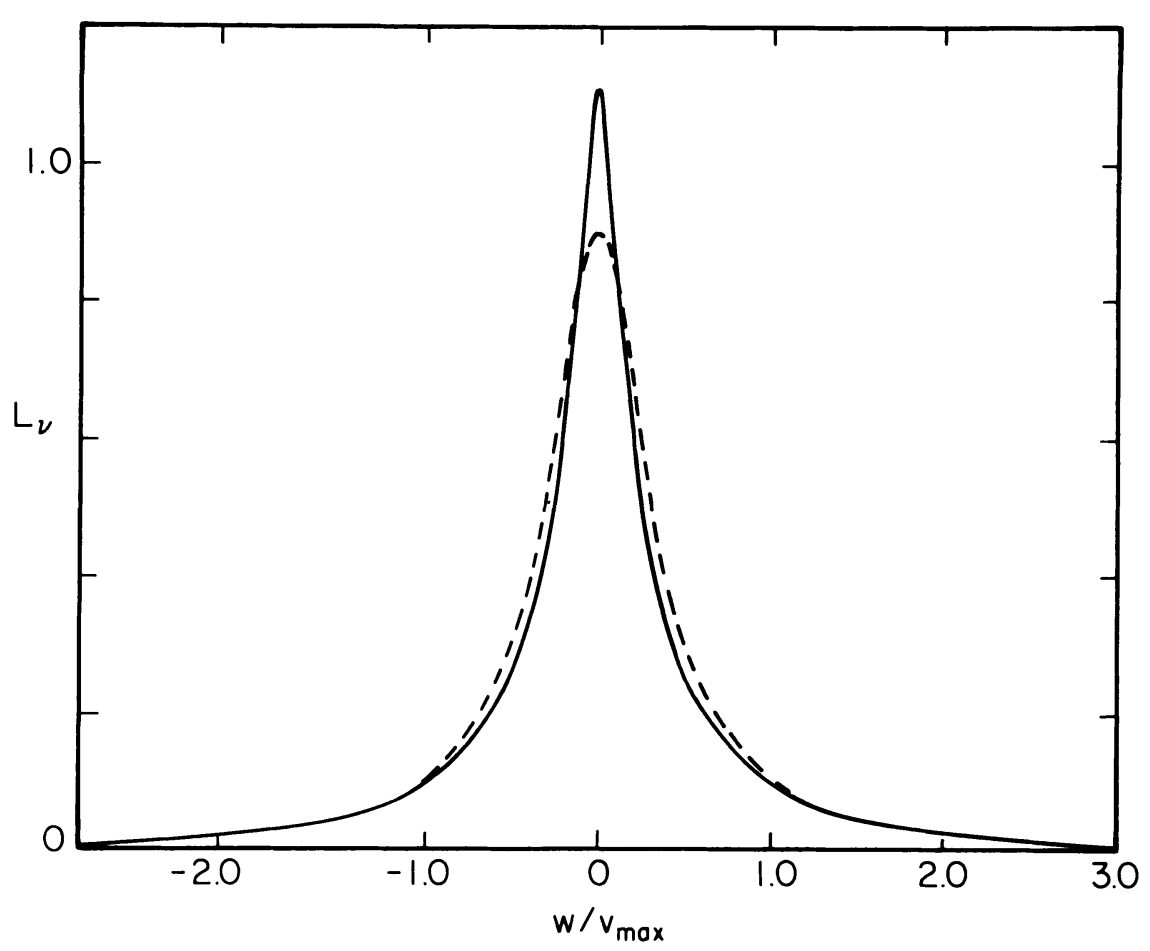

Figure 1. Line profiles for bound tidal debris on highly eccentric orbits with binding energy $\mathrm{E}=-1 / 2 v_{o}^{2}$. Dashed curve is equation (4) for $a=1, \mu=1 / 2$ and $v_{o}=2^{-1 / 2}$ $v_{\max }$, corresponding to the median values of $\mu$ and $v_{o}^{2}$. Solid curve is angle averaged profile, $L_{\nu}=L_{o} \ln \left[1+v_{o}^{2} /\left(w^{2}+v_{\phi}^{2}\right)\right]$, where $v_{o}=2^{-1 / 2} v_{\max }$ and $v_{\phi}=0.1 v_{o}$. Profiles are normalized so that $\int_{-\infty}^{\infty} L_{\nu}(w) d w / v_{\max }=1$.

and they have vertical gravity components $\mathrm{g}_{z} \approx 10^{5}\left(\mathrm{~L} / \mathrm{L}_{E}\right) \mathrm{cm} \mathrm{s}^{-2}$, where we have used a thickness $\mathrm{H} / \mathrm{R} \approx \mathrm{L} / \mathrm{L}_{E}$ for a radiation pressure supported disk. These values suggest that the "UV bump" might be emitted under conditions resembling the atmospheres of O-stars. Kolykhalov and Sunyaev (1984) synthesized thermal disk spectra using published stellar atmospheres and found large Lyman edges in absorption. Such features appear to be rare in QSO spectra (see contribution by Kinney, Antonucci and Ford in this volume). M. Malkan and coworkers (see contribution by Sun in this volume) similarly predict substantial Lyman edges, but they find at least one QSO whose spectrum seems consistent with the disk model prediction.

The Lyman edge is crucial to the disk model, since it provides an opportunity to measure the Doppler velocity of the power producing gas. Increased theoretical and observational efforts thus seem warranted. One suggestion for weakening the Lyman 
edge is to postulate a near balance of vertical gravity and radiation pressure, so that the net effective gravity, $\mathrm{g}_{e f f}=\mathrm{g}_{z}-\mathrm{g}_{r a d}$, is small. The discussion of $\mathrm{g}_{e f f}$ by Kolykhalov and Sunyaev (1984) shows that the entire vertical disk structure must be considered in assessing $\mathrm{g}_{e f f}$. In a thin disk $\mathrm{g}_{z}$ may barely exceed $\mathrm{g}_{\mathrm{rad}}$ near the midplane, where both increase as $z$; but near the surface, $\mathrm{g}_{\text {rad }}$ levels off whereas $\mathrm{g}_{z}$ continues to increase as $z$. Thus, at the photosphere $g_{e f f} \approx g_{z} \approx\left(\mathrm{GM} / R^{2}\right)(z / R)$. In addition to consideration of the disk structure, understanding the thermal emission requires careful model atmosphere work. Efforts described by Laor and by Scott and O'Dell in this volume are valuable beginnings, but non-LTE models may be necessary to predict the Lyman edge accurately. The atmospheric structure may be affected by nonthermal radiation incident from above, and by mechanical heating of the surface layers (Liang and Price 1977). Such careful modeling may also help to settle the question of whether the thermal disk emission contributes to the observed soft X-rays (Czerny and Elvis 1987; Wandel and Petrosian 1988).

Radiation tori may also be a source of thermal emission. Sikora (1981) calculates the beaming of radiation from the funnel of a disk and finds that most of the luminosity emerges in a beam of width comparable with the funnel opening angle. To an observer in the beam, the luminosity would appear to be several times $L_{E}$. The energy distribution of such a torus may also be a candidate for the "UV bump." Madau (1988) explores the aspect dependence of the emergent energy distribution. A definitive model of radiation tori is not at hand, and spectral predictions are now only illustrative. The stability of radiation tori was called into question by Papaloizou and Pringle (1984), who found rapidly growing dynamical instabilities in thick disks. There are a variety of indications of beaming of QSO radiation (for example, the discussion by Miller in this volume), and the structure and stability of radiation tori is an important problem for study.

The polarization of the thermal continuum may be an important test of the disk model. A thin disk has an electron scattering atmosphere in the inner zone, so that a flat disk would give linear polarizations as calculated by Chandrasekhar (1960). This varies from $p=0$ to $p=-11.7 \%$ for observer angles from $\theta=0^{\circ}$ (face on) to $\theta=90^{\circ}$ (edge-on), with $p=-2.3 \%$ for a median inclination $\theta=60^{\circ}$. The negative sign corresponds to an electric vector perpendicular to the disk axis projected onto the sky. In contrast, most Seyfert 1 galaxies and QSOs have linear polarizations of roughly $+1 \%$, where the disk axis is identified with the observed radio axis (Stockman, Moore, and Angel 1984; Stockman, Angel and Miley 1979; Miller, this volume). Webb and Malkan (1987) called attention to this possible difficulty for the disk model.

Coleman and Shields (1988) have explored the effect of local surface irregularities on the polarization of a scattering atmosphere. If the surface undergoes vertical excursions of height comparable with their spacing, perhaps surface waves of some kind, then a significant fraction of the light emitted by one structure will scatter off the slopes of neighboring structures. Coleman and Shields calculated polarizations for a linear ridge-valley geometry and for a geometry of semi-spherical depressions. In each case, positive or negative polarizations of only a few tenths of a percent occur over most viewing angles, and large negative polarizations do not occur at any angle. If the disk emits an essentially unpolarized continuum, then the observed positive polarization might be imposed by scattering in an X-ray heated wind at larger radius (Begelman, McKee, and Shields 1983). Such a picture may be particularly appealing 
if the "nonthermal power law" and thermal disk components are determined to have the same, wavelength independent polarization. The postulated surface irregularities might arise from various causes such as turbulence in the disk or incident nonthermal continuum whose radiation pressure produces an outward shear of the surface layers. The irregularities can occur on any length scale from the electron scattering mean free path, $\ell=(\kappa e s \rho)^{-1}$, in the atmosphere to the disk semithickness $H$.

Coleman and Shields (1988) also calculated the polarization of light emitted from a thick disk, modelled as a cone of half angle $8.6^{\circ}$ imbedded in a sphere with flux injected into the funnel according to Paczynski (1981). Beaming along the cone was found in agreement with Sikora (1981). The polarization was positive for most viewing angles, with a maximum of $+2 \%$. Half the luminosity is emitted inside $\theta=30^{\circ}$, and the polarization at this angle is $+0.5^{\circ}$. These results agree quite well with observed polarizations. The conclusion is that the embarassing negative polarization of a smooth thin disk are avoided by roughened thin disks and also by thick disks.

\subsection{ACCRETION DISK PHYSICS}

The subject of AGN is rich with exotic phenomena observed at all wavelengths from radio to gamma ray. The basic physics of standard thin disks seems rather tame in comparison with some of the topics that AGN theorists love to explore. Nevertheless, the more conservative physics of thermally emitting thin disks may afford the best opportunity to make reliable observational predictions that could establish or rule out the existence of such disks in AGN. The nagging question of viscosity and angular momentum transport is of fundamental importance. Models of dwarf novae (see review by Smak 1984) support the rough validity of the " $\alpha$ model" with $\alpha \approx 10^{-1}$, but AGN disk theory cannot proceed with full confidence without a better understanding of viscosity. A critical part of this will be to settle whether the viscous stress, in regions dominated by radiation pressure, is better approximated by the prescription $w=\alpha\left(P_{\text {rad }}+P_{\text {gas }}\right)$ or $w=\beta P_{g a s}$; this choice greatly affects the stability and optical depth of the "inner" disk zone that may produce the "UV bump." If $P_{r a d}$ is included, then the inner zone is thermally and viscously unstable (Pringle 1981; Lightman and Eardley 1974).

A possible application of "tame" disk physics was considered by Clarke and Shields (1989). This involves applying to AGN disks the hydrogen ionization limit cycle that is a promising theory of dwarf nova outbursts (see review by Smak 1984). At disk radii where the midplane temperature is $\sim 20,000 \mathrm{~K}$, hydrogen is partially ionized. The opacity reaches a peak in this range, as a result of which the vertical structure of the disk has no stable solution for a certain range of surface effective temperatures, $\mathrm{T}_{\text {eff }}$. A corresponding radial zone in the disk undergoes quasiperiodic oscillations between alternative "warm" and "cool" stable states. Based on numerical computations by Clarke (1987), Clarke and Shields find for AGN disks that the oscillations produce strong variations in the thermal continuum of the disk in the 1 to $5 \mu \mathrm{m}$ wavelength band. (The disk surface is cooler than in dwarf novae because the vertical optical depth is larger.) These variations are characterized by a unique period luminosity relation,

$$
P \approx(5 y r)\left(\frac{0.15}{\alpha}\right)\left(\frac{\nu L_{\nu}^{\prime}}{10^{4} L_{\odot} s r^{-1}}\right)^{1 / 2} \nu_{15}^{-2 / 3}
$$


where $L_{\nu}^{\prime}=d L_{\nu} / d \Omega$ is the thermal luminosity emitted normal to the disk per steradian, and $\nu_{15}=\nu / 10^{15} \mathrm{~Hz}$. Variations on as short a timescale as $\sim 10$ years might be observable in the Galactic center and nearby nuclei such as M 81. For more luminous AGN, the variations might be manifested as statistical fluctuations in the infrared continuum of a sample of objects.

\section{Conclusions}

The fundamental question of QSO energy production is most often asked in terms of accretion disks around supermassive black holes. The most immediate hope for observational confirmation may come from the theoretically "tame," and therefore predictable, models of thin disks. Detailed predictions of the behavior of such disks at all radii are needed, preferably incorporating an improved understanding of viscosity. Most promising today is the possibility that the blue-ultraviolet continuum "bump" in AGN spectra may be thermal continuum from the inner region of a disk. Further work on the spectral features, energy distribution, and stability of this region may be richly rewarding. An explanation of the broad lines in terms of tidal debris would confirm the presence of a black hole and the operation of an important accretion source.

\section{References}

Begelman, M.C. 1985, in Astrophysics of Active Galaxies and Quasi-Stellar Objects, ed. J.S. Miller (Univ. Science Books: Mill Valley) p. 411.

Begelman, M.C., McKee, C.F., and Shields, G.A. 1983, Ap. J., 271, 70.

Capriotti, E.R., Folz, C.B., and Byard, P. 1980, Ap. J., 241, 903.

Carroll, T. J., and Kwan, J. 1985, Ap. J., 288, 73.

Chandrasekhar, S. 1960, Radiative Transfer, (Dover: New York).

Clarke, C. J. 1987, Ph.D. Thesis, Oxford Univ.

Clarke, C. J., and Shields, G.A. 1989, Ap. J ., in press.

Coleman, H., and Shields, G. 1988, in preparation.

Czerny, B., and Elvis, M. 1987, Ap. J., 321, 305.

Dressler, A., and Richstone, D.O. 1988, Ap. J., 324, 701.

Fabian, A.C., and Canizares, C.R. 1988, Nature, 333, 829.

Fabricant, D., and Gorenstein, P. 1987, Ap. J., 267, 535.

Ferland, G.J., and Shields, G.A. 1985, in Astrophysics of Active Galaxies and QuasiStellar Objects, ed. J.S. Miller (Univ. Science Books: Mill Valley), p. 157.

Gaskell, C.M. 1988, in Proceedings of the Georgia State University Conference on Active Galactic Nuclei, eds. H. R. Miller and P. J. Wiita (Springer: New York), p.

Gaskell, C.M. and Sparke, L.S. 1986, Ap. J., 306, 175.

Halpern, J. P. and Filippenko, A.V. 1988, Nature, 331, 46. 
Hills, J.G. 1975 Nature, 254, 295.

Hubbard, E. N., and Puetter, R.C. 1983, Ap. J., 265, 35.

Joly, M., Collin-Souffrin, S., Masnou, J. L., and Nottale, L. 1985, Astron. Ap., 152, 282.

Kolykhalov, P.I., and Sunyaev, R.A. 1984, Adv. Space Res., 3, 249.

Kormendy, J. 1988, Ap. J., 325, 128.

Krolik, J.H., and London, R.A. 1983, Ap. J., 267, 18.

Kwan, J., and Carroll, T.J. 1982, Ap. J., 261, 25.

Lacy, J.H., Townes, C.H., and Hollenbach, D.J. 1982, Ap. J., 262, 120.

Liang, E.T.P. and Price, R.H. 1977, Ap. J., 218, 247.

Lightman, A.P., and Eardley, D.M. 1974, Ap. J. (Letters), 187, L1.

Lynden-Bell, D. 1969, Nature, 223, 690.

Malkan, M.A., and Sargent, W.L.W. 1982, Ap. J., 254, 22.

Madau, P. 1988, Ap. J., 327, 116.

Margon, B., Grandi, S.A., and Downes, R.A. 1980, Ap. J., 241, 306.

Mathews, W. G., and Blumenthal, G. R. 1977, Ap. J., 214, 10.

Mathews, W. G., and Capriotti, E. R. 1985, in Astrophysics of Active Galaxies and Quasi-Stellar Objects, ed. J. S. Miller (Univ. Science Books: Mill Valley), p. 185.

McCray, R. 1979, in Active Galactic Nuclei, ed. C.R. Hazard and S. Mitton (Cambridge: Cambridge University Press), p. 227.

Osterbrock, D.E. (1985), in Astrophysics of Active Galaxies and Quasi-Stellar Objects, ed. J.S. Miller (Univ. Science Books: Mill Valley), p. 111.

Paczynski, B. 1981, Mitteilungen der Astronomischen Gesellschaft, 57, 27.

Papaloizou, J.C.B., and Pringle, J.E. 1984, Mon. Not. Roy. Astr. Soc., 208, 721.

Peterson, B.M., Meyers, K.A., Capriotti, E.R., Foltz, C.B., Wilkes, B.J., and Miller, H.R. 1985, Ap. J., 292, 164.

Pringle, J.E. 1981, Ann. Rev. Astr. Ap., 19, 137.

Rees, M. J. 1988, Nature, 333, 523.

Serabyn, E., Lacy, J.H., Townes, C.H., and Bharat, R. 1988, Ap. J., 326, 171.

Shakura, N.I. and Sunyaev, R.A. 1973, Astr. Ap., 254, 22.

Shields, G.A. 1978a, Nature, 272, 706.

Shields, G.A. 1978b, in Pittsburgh Conference on BL Lac Objects, ed. A. M. Wolfe (Pittsburgh: Univ. of Pittsburgh Press), p. 257.

Shields, G.A., and Wheeler, J.C. 1978, Ap. J., 222, 667.

Sikora, M. 1981, Mon. Not. Roy. Astr. Soc., 196, 257. 
Soltan, A. 1982, Mon. Not. Roy. Astr. Soc., 200, 115.

Smak, J. 1984, Publ. Astr. Soc. Pac., 96, 5.

Stockman, H.S., Angel, J.R.P., and Miley, G.K. (1979), Ap. J. (Letters), 227, L55.

Stockman, H.S., Moore, R. L., and Angel, J.R.P. (1984), Ap. J., 279, 485.

Tonry, J. 1984, Ap. J., 203, L27.

Ulrich, M. H., et al. 1984, Mon. Not. Roy. Astr. Soc., 206, 221.

Wandel, A. and Petrosian, V. 1988, Ap. J. (Letters), 329, L11.

Webb, W. and Malkan, M. 1987, in The Physics of Accretion Onto Compact Objects, eds. K.O. Mason, M.G. Watson, and N.E. White (Springer Verlag: Berlin), p. 15. 